\title{
SELEÇÃO DE ÁRVORES MATRIZES DA ESPÉCIE SENEFELDERA VERTICILLATA (VELL.) CROIZAT EM DOIS FRAGMENTOS FLORESTAIS DO SUL DO ESPIRITO SANTO
}

\author{
Alessandra Abreu Rodrigues Vieira ${ }^{1}$ \\ Lucimara Cruz de Souza ${ }^{2}$ \\ Adelson Lemes da Silva Júnior ${ }^{3}$ \\ Kelmer Mozer Moro ${ }^{4}$ \\ Mariana Cruz de Souza ${ }^{5}$ \\ Aléxia Gonçalves Pereira ${ }^{6}$ \\ Marcos Vinicius Winckler Caldeira ${ }^{7}$ \\ Fábio Demolinari de Miranda ${ }^{8}$
}

Resumo: O Brasil abriga a flora mais rica do planeta, sendo a Floresta Atlântica um dos sistemas mais diversificados que compõe essa paisagem. A coleta de sementes de alta qualidade é muito importante para qualquer programa de produção de mudas voltado para restauração e conservação dos recursos genéticos. Dessa forma, com esse estudo objetivou-se avaliar indivíduos de Senefeldera verticillata (Vell.) Croizat presentes na FLONA de Pacotuba e RPPN Cafundó (Cachoeiro de Itapemirim - Espírito Santo), como matrizes para banco de sementes. O maior DAP foi de $17,6 \mathrm{~cm}$ e o menor foi de $5 \mathrm{~cm}$, a média foi de 8,66 $\mathrm{cm}$. Todas as matrizes avaliadas encontravam-se em boas condições de sanidade e aparência fenotípica. A partir dos dados foi possível identificar árvores matrizes que servirão como banco de sementes e empregadas nos projetos de reflorestamento e recuperação de áreas degradadas da própria região.

Palavras-chave: Manejo; Floresta atlântica; Reflorestamento; Banco de sementes.

\footnotetext{
${ }^{1}$ Centro de Ciências Agrárias/Programa de Pós-Graduação em Genética e Melhoramento Universidade Federal do Espírito Santo, Brasil. E-mail: abreuepires@gmail.com.

2 Centro de Ciências Agrárias/Programa de Pós-Graduação em Genética e Melhoramento Universidade Federal do Espírito Santo, Brasil. E-mail: lucimaracruz15@hotmail.com.

${ }^{3}$ Centro de Ciências Agrárias/Programa de Pós-Graduação em Genética e Melhoramento Universidade Federal do Espírito Santo, Brasil. E-mail: adelsonlemes@yahoo.com.br.

${ }^{4}$ Centro de Ciências Agrárias/Programa de Pós-Graduação em Genética e Melhoramento Universidade Federal do Espírito Santo, Brasil. E-mail: kelmer.moro@gmail.com.

${ }^{5}$ Centro de Ciências Agrárias/Programa de Pós-Graduação em Genética e Melhoramento Universidade Federal do Espírito Santo, Brasil. E-mail: scruz.mariana@gmail.com.

${ }^{6}$ Centro de Ciências Agrárias/Programa de Pós-Graduação em Genética e Melhoramento Universidade Federal do Espírito Santo, Brasil. E-mail: alexiagp@gmail.com.

7 Centro de Ciências Agrárias/Programa de Pós-Graduação em Genética e Melhoramento Universidade Federal do Espírito Santo, Brasil. E-mail: mvwcaldeira@gmail.com.

${ }^{8}$ Centro de Ciências Agrárias/Programa de Pós-Graduação em Genética e Melhoramento Universidade Federal do Espírito Santo, Brasil. E-mail: fademolinari@yahoo.com.br.
} 